\title{
Excitons Diffusion Length, Tree Dimensionless Numbers and Mean Temperature Dependence of Semiconductor Performance Including Excitons
}

\author{
Modou Faye ${ }^{1, ~ *}$, Ousmane $\operatorname{Ngom}^{1}$, Saliou Ndiaye ${ }^{1}$, Cheikh Mbow ${ }^{2}$, Bassirou Ba ${ }^{1}$ \\ ${ }^{1}$ Department of Physics, Laboratory of Semiconductors and Solar Energy, Faculty of Science and Technology University Cheikh Anta DIOP, \\ Dakar, Senegal \\ ${ }^{2}$ Department of Physics, Laboratory of Fluid Mechanics, Hydraulics and Transfers, Faculty of Science and Technology University Cheikh Anta \\ DIOP, Dakar, Senegal
}

Email address:

Fayendiouma80@gmail.com (M. Faye)

${ }^{*}$ Corresponding author

\section{To cite this article:}

Modou Faye, Ousmane Ngom, Saliou Ndiaye, Cheikh Mbow, Bassirou Ba. Excitons Diffusion Length, Tree Dimensionless Numbers and Mean Temperature Dependence of Semiconductor Performance Including Excitons. International Journal of Materials Science and Applications. Vol. 9, No. 2, 2020, pp. 25-33. doi: 10.11648/j.ijmsa.20200902.11

Received: March 27, 2020; Accepted: April 20, 2020; Published: May 18, 2020

\begin{abstract}
The author, taking into account the non-uniformity of dissociation, the recombination of excitons in the depletion region, as well as the variability of the coefficients as a function of temperature, used dimensional analysis. Thus, by grouping together the physical parameters, dependent and independent variables, he generates dimensionless numbers. Among the latter, we have the ratio between the diffusion time and the lifetime of the charge carriers (Fourier number); the ratio between the imposed heat flux and that thermal conduction (heating factor) and the ratio between the mobility of the excitons and that of the electrons. The motivation of the author is on the one hand to show the influence of these dimensionless numbers on the diffusion lengths of the charge carriers and on the other hand their influence and that of the diffusion lengths on the total photocurrent density of the carriers. Therefore, he studied the effects of the mean temperature and those of the mobility ratio on the total density of the photocurrent. In order to carry out such work, the author opted for the finite volume method combined with an iterative line-by-line relaxation method of the Gauss-Seidel type as a method of solving his physical problem.
\end{abstract}

Keywords: Diffusion Lengths, Adimensional Numbers, Mobility, Excitons

\section{Introduction}

In this article we study the total photocurrent density of the carriers by considering two cases. In the first case we consider a constant excitation diffusion length. In other words, the diffusion coefficient and the lifetime of the excitons are taken constant. In the second case, it varies as a function of the mean temperature obtained from the general heat equation without source term. In both cases, the diffusion length of the electrons varies as a function of the average temperature. In this study we have as a physical model a silicon-based semiconductor, in static regime under monochromatic illumination, in the presence of excitons, including the depletion region. Some authors, such as $\mathrm{M}$. Burgelman and B. Minnaer [1], consider the base to be the region generating most of the photocurrent density. As well as, Zh.
Karazhanov [2] has studied the depletion region, under darkness, while neglecting the electric field in this region. He studied the effect of temperature on the diffusion length of carriers. However, most of the work of some authors is limited to analytical treatment in the p-doped region, assuming that exciton dissociation and recombination are uniform in the volume of this region [1-4]. In these models, the coupling effects between electrons and excitons are considered either neglected or linear and the phenomenological coefficients are taken constant.

M. Faye and others have developed a numerical model applicable to inorganic semiconductors in the presence of excitons by including the depletion region [5]. They have shown that the generation of minority carriers (electrons and excitons) depends on the absorption and the mean temperature associated with the volume coupling coefficient 
$b=\left(10^{-2} \times T_{\text {moy }}^{-2}+2.5 \times 10^{-6} \times T_{\text {moy }}^{-0.5}+1.5 \times 10^{-7}\right)$ They have also shown that absorption can be dominated by electrons $\left(f_{e}=1\right)$ or excitons $\left(f_{x}=1\right)$ [6]. They have developed other numerical models [7-11]. In all these models, they have taken into account the non-uniformity of dissociation, the recombination of excitons in the depletion region. They have always considered the variability of the coefficients as a function of temperature except for the exciton diffusion coefficient.

In this study we numerically analyze the role of three dimensionless characteristic quantities. The importance of this study is mainly due to the fact that we have considered the diffusion length of the electrons and possibly that of the variable excitons. In addition, we have considered a wider range of the mean temperature. The non-uniformity of dissociation and recombination of excitons in the depletion region, as well as the variability of the coefficients as a function of temperature are always taken into account.

\section{Physical Model}

We consider a semiconductor of length L [5], of one-dimensional character, with non-homogeneously doped regions. They are conductive. The $\mathrm{n}^{+}$type region, called emitter, is heavily doped with $\mathrm{N}_{D}=10^{+19} \mathrm{~cm}^{-3}$. Our study is limited in the depletion region and in the base doped at $\mathrm{N}_{\mathrm{A}}=10^{+16} \mathrm{~cm}^{-3}$. The electric field in this zone is a linear function of the abscissa $\mathrm{z}$ which can be put in the form: $E(z)=\frac{E_{m}}{w}(w-z)$ and $b(z)=b[E(z)]$ in $(0 \leq z \leq w)$.

The thickness of the space load zone is considered variable. It depends on the mean temperature. Therefore, the electric field also depends on the mean temperature. The bound or free electron-hole pairs from the incident photons are dissociated in the depletion region by the electric field.

\section{Mathematical Formulation}

When our physics is subjected to illumination, it governs, neglecting the terms of inertia, the diffusion equations of the load carriers. They are written as follows:

$$
\begin{aligned}
& \frac{\partial}{\partial z}\left\{D_{e} \frac{\partial n_{e}}{\partial z}\right\}+\frac{E_{m} \mu_{e}}{w} \cdot \frac{\partial}{\partial z}\left\{n_{e}(w-z)\right\}=\frac{1}{\tau_{e}} \cdot \frac{n_{e} n_{h}-n_{i n}^{2}}{n_{e}+n_{h}+2 n_{i n}}+b\left(n_{e} n_{h}-n_{x} n^{\circ}\right)-f_{e} G_{e h} \\
& \frac{\partial}{\partial z}\left\{D_{x} \frac{\partial n_{x}}{\partial z}\right\}=\frac{n_{x}-n_{x 0}}{\tau_{x}}-b\left(n_{e} n_{h}-n_{x} n^{\circ}\right)-f_{x} G_{x} \\
& \left\{\begin{array}{l}
z=0 \Rightarrow n_{e}(0)=N_{D} \\
z=L \Rightarrow \frac{\partial}{\partial z}\left\{D_{e} n_{e}\right\}=-S_{e}\left[n_{e}(L)-n_{e 0}\right]+b_{s}\left[n_{x}(L)-n_{x 1}\right]
\end{array}\right.
\end{aligned}
$$

\subsection{For the Electrons} expression is given by:

$$
b v\{E(z)\}=b v_{-} l o w \times \exp \left[\left(1-\frac{z}{w}\right) \times \ln \left(\frac{b v_{-} \max }{b v_{-} l o w}\right)\right]
$$

With,

$$
\text { bv_low }=10^{-16} \mathrm{~cm}^{3} \mathrm{~s}^{-1}
$$

$10^{-16} \mathrm{~cm}^{3} \mathrm{~s}^{-1} \leq b v_{-} \max \leq 10^{-7} \mathrm{~cm}^{3} \mathrm{~s}^{-1}$

It depends on the dissociation field of the excitons and the mean temperature of the semiconductor. Other parameters such as the diffusion coefficients of the electrons and excitons are also function of the mean temperature of the semiconductor. They are given by Einstein's relation [12]. They are possibly dependent on the mean temperature.

These equations are not only coupled, but they contain phenomenological coefficients that depend on the temperature field, which can only be understood by solving the general thermal diffusion equation without a source term.

$$
\frac{\partial T}{\partial t}=a \frac{\partial^{2} T}{\partial z^{2}}
$$

\subsection{For the Excitons}

$$
\left\{\begin{array}{l}
z=0 \Rightarrow \frac{\partial}{\partial z}\left\{D_{x} n_{x}\right\}=S_{x 0}\left[n_{x}(0)-n_{x 0}\right]-b_{s}\left[n_{x}(0)-n_{x 1}\right] \\
z=L \Rightarrow \frac{\partial}{\partial z}\left\{D_{x} n_{x}\right\}=-S_{x}\left[n_{x}(L)-n_{x 0}\right]-b_{s}\left[n_{x}(L)-n_{x 1}\right]
\end{array}\right.
$$

\subsection{Conditions on the Temperature}

$$
\begin{cases}t=0 & \Rightarrow T(z, 0)=0 \\ z=0 & \Rightarrow q=-\lambda \frac{\partial T}{\partial z}=q_{m} g(t) \\ z=L & \Rightarrow \frac{\partial T}{\partial z}=0\end{cases}
$$

Mixed boundary conditions reveal a very important physical parameter, called the rate of conversion of excitons into free electrons. In this model, it is $b s=10^{+7} \mathrm{~cm} \mathrm{~s}^{-1}$.

The dimensionless equations of a coupled system and that of heat become:

The resulting system is finally closed by mixed boundary

$$
F_{e 0} \frac{\partial}{\partial z^{*}}\left\{D_{T}^{*} \frac{\partial n_{e}^{*}}{\partial z^{*}}\right\}+A \frac{\partial}{\partial z^{*}}\left\{n_{e}^{*}\left(w^{*}-z^{*}\right)\right\}=\frac{n_{e}^{*} n_{h}^{*}-n_{i n}^{*}{ }^{2}}{n_{e}^{*}+n_{h}^{*}+2 n_{i n}^{*}}+B_{e}\left(n_{e}^{*} n_{h}^{*}-n_{x}^{*} n_{1}^{*}\right)-C_{e} f_{e} G^{*}
$$




$$
F_{x 0} \frac{\partial}{\partial z^{*}}\left\{R_{\mu} D_{T}^{*} \frac{\partial n_{x}^{*}}{\partial z^{*}}\right\}=\left(n_{x}^{*}-n_{x 0}^{*}\right)-B_{x}\left(n_{e}^{*} n_{h}^{*}-n_{x}^{*} n_{1}^{*}\right)-C_{x}\left(1-f_{e}\right) G^{*}
$$

$$
\frac{\partial T^{*}}{\partial t^{*}}=\frac{\partial^{2} T^{*}}{\partial z^{* 2}}
$$

These dimensionless equations were obtained from the modelling assumptions and the physical reference quantities [5].

And the characteristic dimensionless numbers are:

$F_{0}=\frac{\tau \times D^{0}}{L^{2}}$, is the ratio between diffusion time and

lifetime (Fourier number). The quantity $D^{0}$ is the electron diffusion coefficient calculated from the ambient temperature $T_{a}$ which is considered constant.

The quantity $F a c t-c h=\frac{\Delta T_{r}^{*}}{T_{a}}$, is called the heating factor.

It is the ratio between the imposed heat flux and that thermal conduction.

The quantity $R_{\mu}=\frac{\mu_{x}}{\mu_{e}}$, is the ratio between the mobility of the excitons and that of the electrons.

To complete system (7), (8) and (9) in the interval [0, 1], we associate initial conditions and dimensionless boundary conditions with it:

\subsection{For the Electrons}

$$
\left\{\begin{array}{l}
z^{*}=0 \Rightarrow n_{e}^{*}(0)=N_{D}^{*} \\
z^{*}=1 \Rightarrow A_{L e} \frac{\partial}{\partial z^{*}}\left\{D_{T}^{*} n_{e}^{*}\right\}_{z=1}=-\left[n_{e}^{*}(1)-n_{e 0}^{*}\right]+B_{L e}\left[n_{x}^{*}(1)-n_{x 1}^{*}\right]
\end{array}\right.
$$

\subsection{For the Excitons}

$$
\left\{\begin{array}{l}
z^{*}=0 \Rightarrow A_{0 x} \frac{\partial}{\partial z^{*}}\left\{R_{\mu} D_{T}^{*} n_{x}^{*}\right\}_{z=0}=\left[n_{x}^{*}(0)-n_{x 0}^{*}\right]-B_{0 x}\left[n_{x}^{*}(0)-n_{x 1}^{*}\right] \\
z^{*}=1 \Rightarrow A_{L x} \frac{\partial}{\partial z^{*}}\left\{R_{\mu} D_{T}^{*} n_{x}^{*}\right\}_{z=1}=-\left[n_{x}^{*}(1)-n_{x 0}^{*}\right]-B_{L x}\left[n_{x}^{*}(1)-n_{x 1}^{*}\right]
\end{array}\right.
$$

\subsection{Conditions on the Temperature}

$$
\left\{\begin{array}{l}
t^{*}=0 \Rightarrow T^{*}\left(z^{*}, 0\right)=0 \\
z^{*}=0 \Rightarrow \frac{\partial T^{*}}{\partial z^{*}}=-g\left(t^{*}\right) \\
z^{*}=1 \Rightarrow \frac{\partial T^{*}}{\partial z^{*}}=0
\end{array}\right.
$$

\section{Digital Model [5]}

Prior to the numerical procedure, the mathematical formulation must be transformed by means of a discretization process into an easy format. It is spatially variable in pitch and tight at the interfaces of the different areas of the domain because of the strong gradients in these regions.

The equations are then integrated into the numerical domain using the finite volume method and the coefficients are approximated by Patankar's power law scheme. The resulting system of algebraic equations is solved using the double-path method combined with an iterative line-by-line relaxation method of the Gauss-Seidel type [13-14].

\section{Results and Discussion}

We present essentially the influences of the three dimensionless numbers on the total photocurrent density of electrons and excitons. We also study the effect of the mean temperature on the diffusion length of electrons and excitons.

Under the assumption of a non-negligible electric field, the photocurrent of the electrons and that of the excitons are given by the sums of two contributions: the diffusion currents and those of the conductions.

$$
\begin{gathered}
J_{e}=q\left\{D_{e} \frac{\partial n_{e}}{\partial z}\right\}+q \cdot n_{e} \cdot \frac{E_{m} \mu_{e}}{w}(\omega-z) \\
J_{x}=q\left\{D_{x} \frac{\partial n_{x}}{\partial z}\right\}+q \cdot n_{x} \cdot \frac{E_{m} \mu_{x}}{w}(\omega-z)
\end{gathered}
$$

The expression of the total photocurrent density is the equation (15):

$$
J=J_{x}+J_{e}
$$

\subsection{First Case: Exciton Diffusion Length $L_{x}=3.43 \cdot 10^{-2} \mathrm{~cm}$, Taken Constant}

\subsubsection{Electron Diffusion Length}

The electron diffusion length $L_{e}$ characterizes the distance that photogenerated electrons can reach before recombining.

a. Influence of the ratio between diffusion time and charge carrier lifetime.

In Figure 1, we represent the electron diffusion length curve obtained by varying the mean temperature for different Fourier number values.

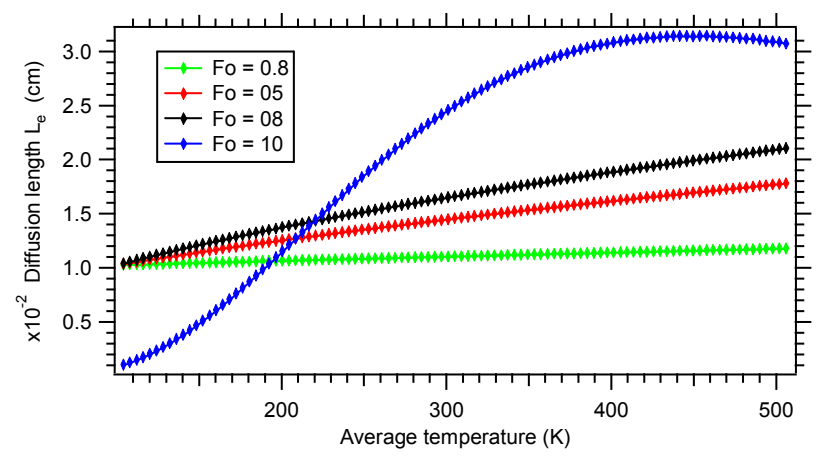

Figure 1. Electron diffusion length as a function of mean temperature for different values of $\mathrm{Fo}$.

$\mathrm{n}_{\mathrm{i}}=1.4510^{10} \mathrm{~cm}^{-3 ;} \mathrm{n} \_$mott $=1.0310^{18} \mathrm{~cm}^{-3 ;} \mathrm{Se}=\mathrm{Sx}=10 \mathrm{~cm} \mathrm{~s}^{-1} ; \mathrm{bv} \_\max =10^{-7} \mathrm{~cm}^{3}$ $\mathrm{s}^{-1} ;$ Fact_ch $=2.10^{-2} ; \overline{\mathrm{L}}_{\mathrm{x}}=3.43 .10^{-2} \mathrm{~cm} ; \mathrm{L}_{\mathrm{e}}=\mathrm{f}$ (mean temperature). 
The diffusion length of the electrons is very sensitive to the variation of the mean temperature, especially for large Fourier number values. It is more sensitive for a higher or equal mean temperature $200 \mathrm{~K}$.

b. Influence of the ratio between imposed heat flux and that of thermal conduction.

We reproduce in figures 2 for $\mathrm{Fo}=0.8$ and $3 \mathrm{Fo}=10$ the variation of the electron diffusion length as a function of the mean temperature for different values of the heating factor.

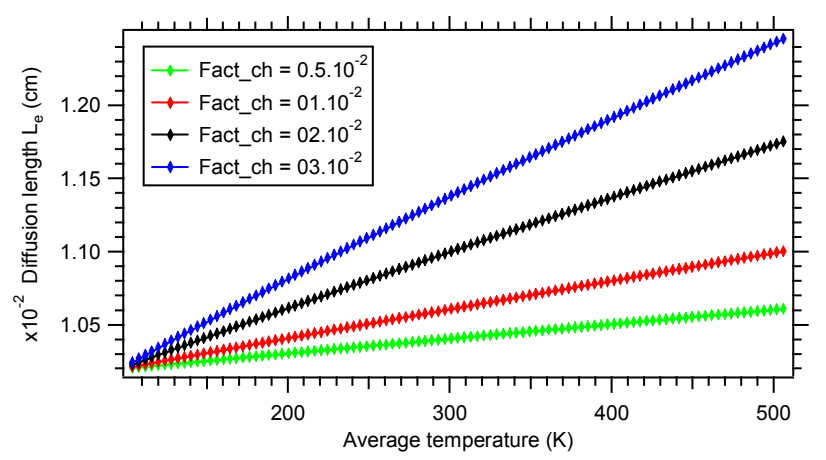

Figure 2. Electron diffusion length as a function of mean temperature for different heating factor values.

$\mathrm{n}_{\mathrm{i}}=1.4510^{10} \mathrm{~cm}^{-3 ;} \mathrm{n} \_$mott $=1.0310^{18} \mathrm{~cm}^{-3 ;} \mathrm{Se}=\mathrm{Sx}=10 \mathrm{~cm} \mathrm{~s}^{-1} ; \mathrm{bv} \_\max =10^{-7} \mathrm{~cm}^{3}$ $\mathrm{s}^{-1} ; \mathrm{L}_{\mathrm{x}}=3 \cdot 43 \cdot 10^{-2} \mathrm{~cm} ; \mathrm{L}_{\mathrm{e}}=\mathrm{f}$ (mean temperature); $\mathrm{Fo}=0,8$.

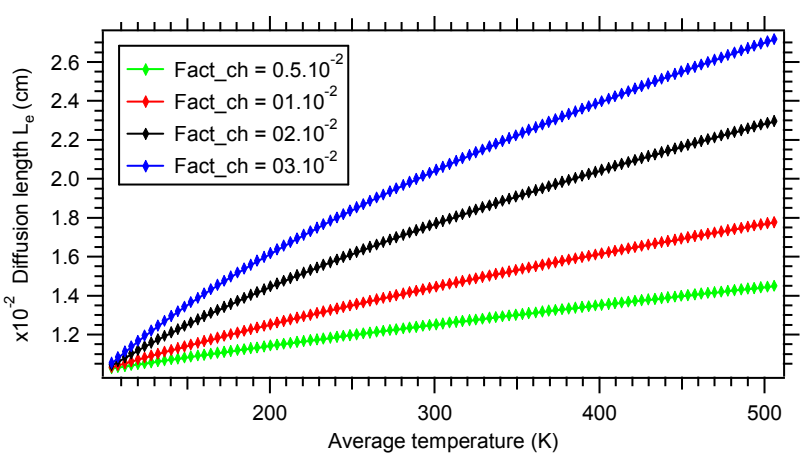

Figure 3. Electron s diffusion length as a function of mean temperature for different heating factor values.

$\mathrm{n}_{\mathrm{i}}=1.4510^{10} \mathrm{~cm}^{-3 ;} \mathrm{n} \_$mott $=1.0310^{18} \mathrm{~cm}^{-3 ;} \mathrm{Se}=\mathrm{Sx}=10 \mathrm{~cm} \mathrm{~s}^{-1} ; \mathrm{bv} \_\max =10^{-7} \mathrm{~cm}^{3}$ $\mathrm{s}^{-1} ; \mathrm{L}_{\mathrm{x}}=3.43 .10^{-2} \mathrm{~cm} ; \mathrm{L}_{\mathrm{e}}=\mathrm{f}$ (mean temperature); $\mathrm{Fo}=10$.

In this section we have the same findings as in Figure 1. In other words, the heating factor has the same effects as the Fourier number on the variation of the electron diffusion length. For a larger Fourier number, there is an augmentation of the diffusion length of the electrons. Only for a mean temperature of $100 \mathrm{~K}$, we have almost the same value of the electron diffusion length whatever the value of the heating factor in each of these figures.

c. Influence of the ratio between the mobility of the excitons and the mobility of the electrons.

In figure 4, we have represented the evolution of the electron diffusion length as a function of the mean temperature for different values of the mobility ratio.

Figure 4 illustrates the negligible effect of the ratio of mobilities to the diffusion length of the electrons.
The observations made on the effect of the mean temperature on the variation of the electron diffusion length of these different figures will help us on the comments of the different profiles of the total photocurrent density of the charge carriers.

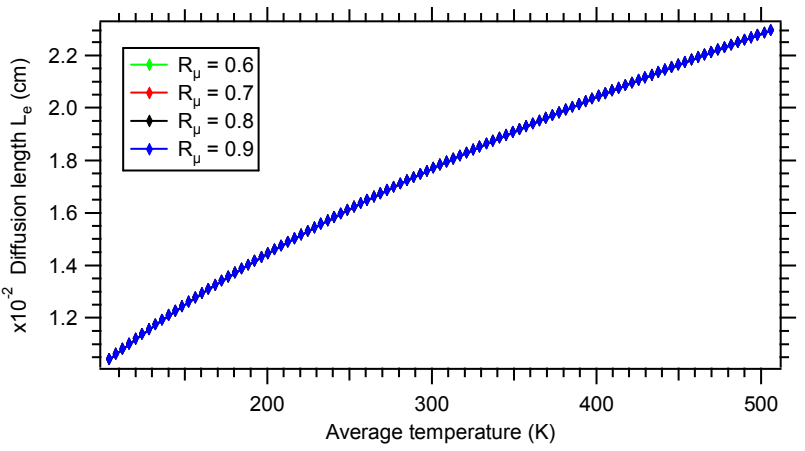

Figure 4. Electron diffusion length as a function of mean temperature for different values of $R \mu$.

$\mathrm{n}_{\mathrm{i}}=1.4510^{10} \mathrm{~cm}^{-3 ;} \mathrm{n} \operatorname{mott}=1.0310^{18} \mathrm{~cm}^{-3 ;} \mathrm{Se}=\mathrm{Sx}=10 \mathrm{~cm} \mathrm{~s}^{-1} ; \mathrm{bv} \max =10^{-7} \mathrm{~cm}^{3}$ $\mathrm{s}^{-1}$; Fact_ch $=2.10^{-2} ; \mathrm{L}_{\mathrm{x}}=3.43 .10^{-2} \mathrm{~cm} ; \mathrm{L}_{\mathrm{e}}=\mathrm{f}$ (mean temperature); $\mathrm{Fo}=10$.

\subsubsection{Total Photocurrent Density as a Function of Temperature}

The curves of total photocurrent density versus mean temperature for an exciton diffusion length $L_{x}=3.43 .10^{-2} \mathrm{~cm}$ are shown in Figures 5, 6, 7 and 8.

a. Influence of the ration between diffusion time and charge carrier lifetime.

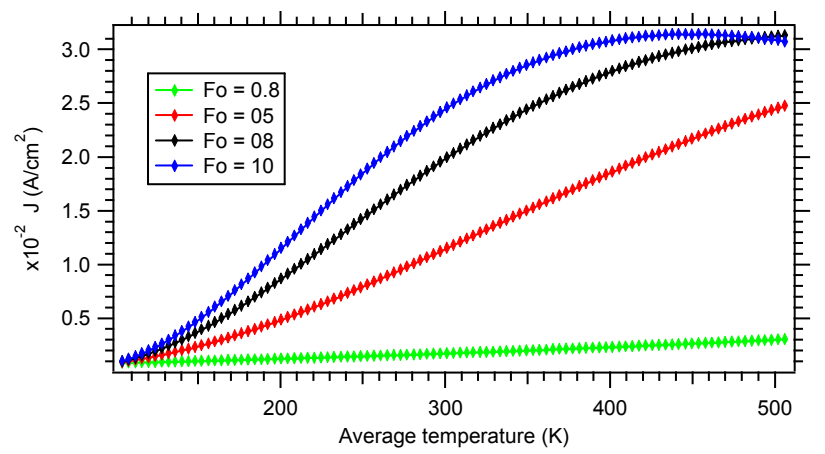

Figure 5. Total photocurrent density of charge carriers as a function of mean temperature for different values of $F$.

$\mathrm{n}_{\mathrm{i}}=1.4510^{10} \mathrm{~cm}^{-3 ;} \mathrm{n} \_$mott $=1.0310^{18} \mathrm{~cm}^{-3 ;} \mathrm{Se}=\mathrm{Sx}=10 \mathrm{~cm} \mathrm{~s}^{-1} ;$ bv_max $=10^{-7} \mathrm{~cm}^{3}$ $\mathrm{s}^{-1} ;$ Fact $\_$ch $=2.10^{-2} ; \overline{\mathrm{L}}_{\mathrm{x}}=3.43 .10^{-2} \mathrm{~cm} ; \mathrm{L}_{\mathrm{e}}=\mathrm{f}$ (mean temperature).

The curves in Figure 5 show that the Fourier number plays a significant role in the distribution of charge carriers; in particular on the variation of the total photocurrent density. As the Fourier number increases, the total photocurrent density of the charge carriers also increases. From the kinematic point of view this magnitude compares the duration of the diffusion phenomenon and the lifetime of the charge carriers. Indeed, an augmentation of Fourier number, for a given semiconductor, is equivalent to an augmentation of $D^{0}$ or $\tau$. So, the combination of these two effects results concomitantly in an augmentation of the total photocurrent density. Numerical simulations have shown us that the diffusion length of 
excitons $L_{x}=3.43 .10^{-2} \mathrm{~cm}$ is greater than that of electrons (see Figure 1) for these different values of the furnace number, hence the significant effect of the latter on the excitation density. Moreover, the augmentation of the density of excitons only reinforces the augmentation of the total photocurrent density. But we note a particular case, a decrease of the total photocurrent density for $\mathrm{Fo}_{\mathrm{O}}=08$ and $\mathrm{Fo}_{\mathrm{O}}=10$. With these Fourier number values, we have an augmentation of the electron density and the excitation density. Beside we have an augmentation of the diffusion length of the electrons, it sends that of the excitons $L_{x}=3.43 .10^{-2} \mathrm{~cm}$. On the other hand, if the density of the excitons increases and if the created exciton has to pass through the whole thickness of the semiconductor to reach the dissociation site without variation of its diffusion length; this can cause a low rate of dissociation of the excitons.

b. Influence of the ratio between imposed heat flux and that of thermal conduction.

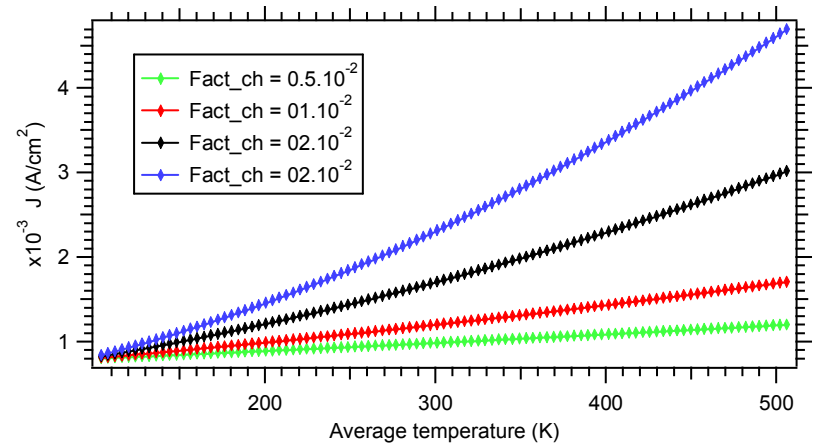

Figure 6. Total photocurrent density of charge carriers as a function of mean temperature for different heating factor values.

$\mathrm{n}_{\mathrm{i}}=1.4510^{10} \mathrm{~cm}^{-3 ;} \mathrm{n} \_$mott $=1.0310^{18} \mathrm{~cm}^{-3 ;} \mathrm{Se}=\mathrm{Sx}=10 \mathrm{~cm} \mathrm{~s}^{-1} ;$ bv_max $=10^{-7} \mathrm{~cm}^{3}$ $\mathrm{s}^{-1} ; \mathrm{L}_{\mathrm{x}}=3 \cdot 43 \cdot 10^{-2} \mathrm{~cm} ; \mathrm{L}_{\mathrm{e}}=\mathrm{f}$ (mean temperature); $\mathrm{Fo}=0,8$.

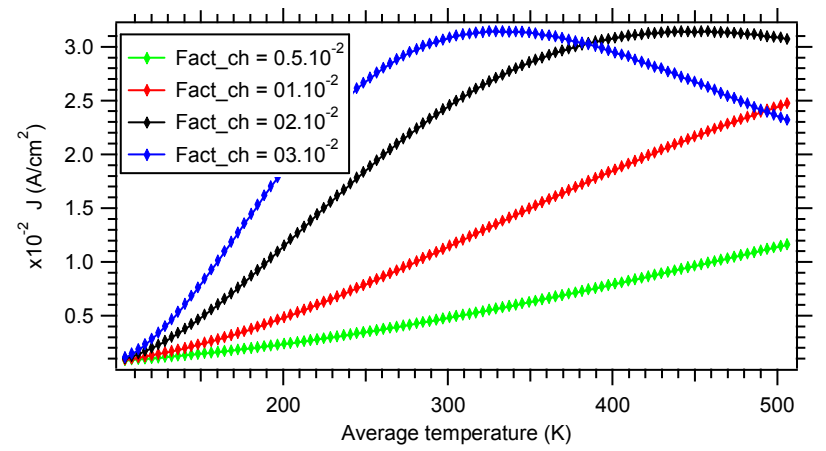

Figure 7. Total photocurrent density of charge carriers as a function of mean temperature for different heating factor values.

$\mathrm{n}_{\mathrm{i}}=1.4510^{10} \mathrm{~cm}^{-3 ;} \mathrm{n} \operatorname{mott}=1.0310^{18} \mathrm{~cm}^{-3 ;} \mathrm{Se}=\mathrm{Sx}=10 \mathrm{~cm} \mathrm{~s}^{-1} ; \mathrm{bv} \max =10^{-7} \mathrm{~cm}^{3}$ $\mathrm{s}^{-1} ; \mathrm{L}_{\mathrm{x}}=3.43 \cdot 10^{-2} \mathrm{~cm} ; \mathrm{L}_{\mathrm{e}}=\mathrm{f}$ (mean temperature); $\mathrm{Fo}_{\mathrm{O}}=10$.

Considering any of the curves in Figures 6 and 7, there is an augmentation of the total photocurrent density of the semiconductor as a function of the mean temperature. This augmentation is more significant with higher values of the heating factor [6-10]. On the other hand, for heating factor values Fact_ch $=2.10^{-2}$ and Fact_ch $=3.10^{-2}$; for higher mean temperatures $350 \mathrm{~K}$ we have a decrease of the total photocurrent density. This decrease can be said to a dissociation of the excitons away from the depletion region in the base where the charge carriers end up recombining.

c. Influence of the ratio between the mobility of the excitons and that of the electrons.

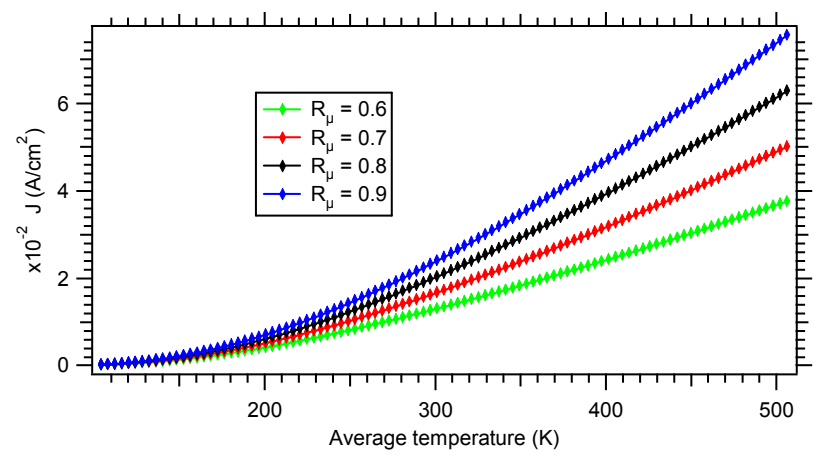

Figure 8. Total photocurrent density of charge carriers as a function of mean temperature for different values of $R \mu$.

$\mathrm{n}_{\mathrm{i}}=1.4510^{10} \mathrm{~cm}^{-3 ;} \mathrm{n} \operatorname{mott}=1.0310^{18} \mathrm{~cm}^{-3 ;} \mathrm{Se}=\mathrm{Sx}=10 \mathrm{~cm} \mathrm{~s}^{-1} ; \mathrm{bv} \max =10^{-7} \mathrm{~cm}^{3}$ $\mathrm{s}^{-1} ;$ Fact_ch $=2.10^{-2} ; \overline{\mathrm{L}}_{\mathrm{x}}=3.43 .10^{-2} \mathrm{~cm} ; \mathrm{L}_{\mathrm{e}}=\mathrm{f}$ (mean temperature); $\mathrm{Fo}=10$.

Figure 8 shows the evolution of the total photocurrent density of the charge carriers as a function of the mean temperature. The positive effect of the mobility ratio on the variation of the total photocurrent density is due to that of the mobility of the excitons. We recall that the mobility ratio compares the mobility of excitons and the mobility of electrons. Since free electrons and excitons interact, by increasing the mobility of excitons, we tend to relaxations phenomena to pass into excited states thus forming other excitons. In other words, the increased mobility of excitons leads to increased mobility of excitons [15]. On the other hand the augmentation of the total photocurrent density is not due to the variation in the diffusion length of the excitons because the mobility ratio has no significant effect on the latter. It is therefore due to the dissociation of the excitons by the electric field following the augmentation of the mean temperature.

\subsection{Second Case: Exciton Diffusion Length Varies as A Function of Mean Temperature}

\subsubsection{Electron and Exciton Diffusion Lengths}

We reproduce in figures $9,10,11,12,13,14,15$ and 16 the variations of the electron and exciton diffusion length as a function of the mean temperature.

a. Influence of the ratio between diffusion time and charge carrier lifetime.

The curves in Figures 9 and 10 show that the effects of Fourier number on the variation of the diffusion length of the charge carriers are antonymous. These effects are positive and negative respectively on the variation of the diffusion length of electrons and excitons. These effects are more sensitive with large Fourier number values. We also note a decrease in the exciton diffusion length as a function of the mean temperature. 


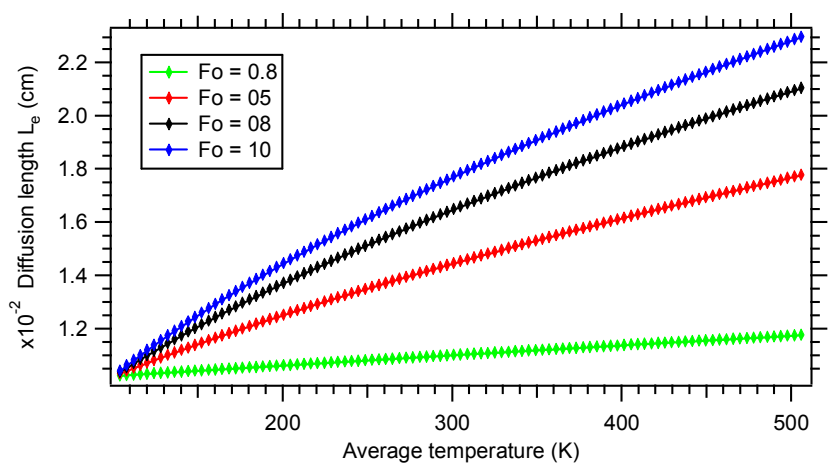

Figure 9. Electron diffusion length as a function of mean temperature for different values of $F o$.

$\mathrm{n}_{\mathrm{i}}=1.4510^{10} \mathrm{~cm}^{-3 ;} \mathrm{n} \_$mott $=1.0310^{18} \mathrm{~cm}^{-3 ;} \mathrm{Se}=\mathrm{Sx}=10 \mathrm{~cm} \mathrm{~s}^{-1} ; \mathrm{bv} \_\max =10^{-7} \mathrm{~cm}^{3}$ $\mathrm{s}^{-1} ;$ Fact_ch $=2.10^{-2} ; \overline{\mathrm{L}}_{\mathrm{e}}=\mathrm{f}$ (mean temperature); $\mathrm{L}_{\mathrm{x}}=\mathrm{f}$ (mean temperature).

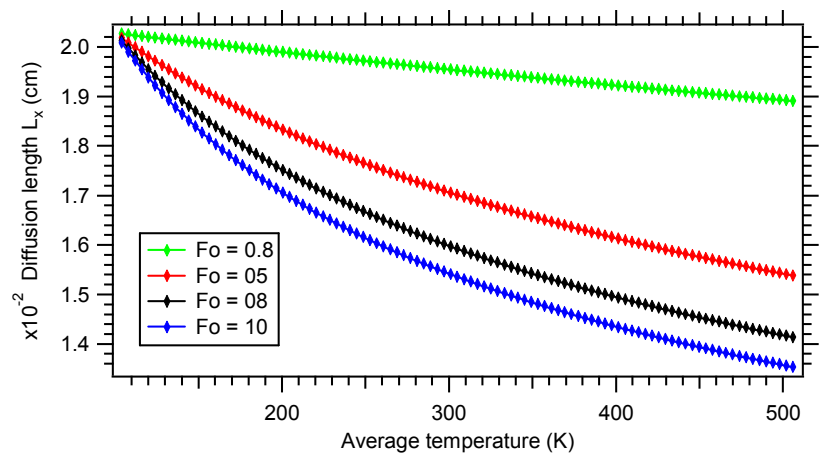

Figure 10. Exciton diffusion length as a function of mean temperature for different values of Fo.

$\mathrm{n}_{\mathrm{i}}=1.4510^{10} \mathrm{~cm}^{-3 ;} \mathrm{n} \_$mott $=1.0310^{18} \mathrm{~cm}^{-3 ;} \mathrm{Se}=\mathrm{Sx}=10 \mathrm{~cm} \mathrm{~s}^{-1} ; \mathrm{bv} \_\max =10^{-7} \mathrm{~cm}^{3}$ $\mathrm{s}^{-1}$; Fact_ch $=2.10^{-2} ; \overline{\mathrm{L}}_{\mathrm{e}}=\mathrm{f}$ (mean temperature); $\mathrm{L}_{\mathrm{x}}=\mathrm{f}$ (mean temperature).

\section{b. Influence of the ratio between imposed heat flux and that of thermal conduction.}

The findings on the variation of electron diffusion length as a function of mean temperature in Figures 2 and 3 remain the same for Figures 11 and 12.

Curves 13 and 14 show that, the effects of the heating factor on the variation of the diffusion length of the excitons are synonymous with those of the Fourier number.

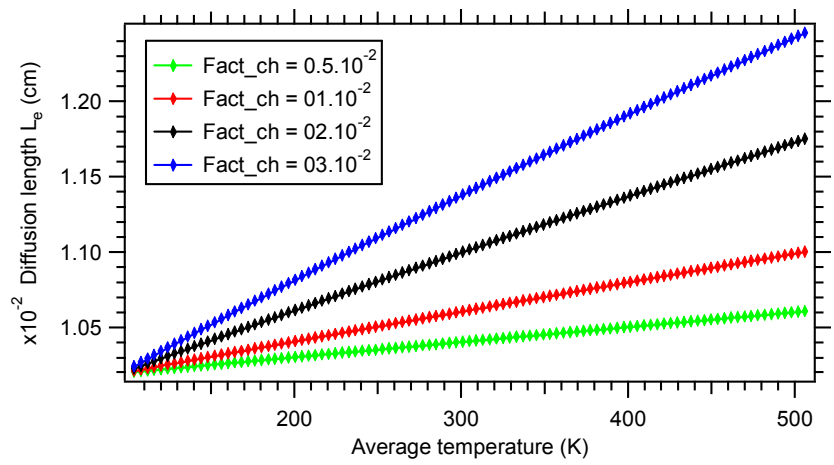

Figure 11. Electron diffusion length as a function of mean temperature for different heating factor values.

$\mathrm{n}_{\mathrm{i}}=1.4510^{10} \mathrm{~cm}^{-3 ;} \mathrm{n} \_$mott $=1.0310^{18} \mathrm{~cm}^{-3 ;} \mathrm{Se}=\mathrm{Sx}=10 \mathrm{~cm} \mathrm{~s}^{-1} ; \mathrm{bv} \max =10^{-7} \mathrm{~cm}^{3}$ $\mathrm{s}^{-1} ; \mathrm{L}_{\mathrm{e}}=\mathrm{f}$ (mean temperature); $\mathrm{Fo}_{\mathrm{o}}=0,8$.

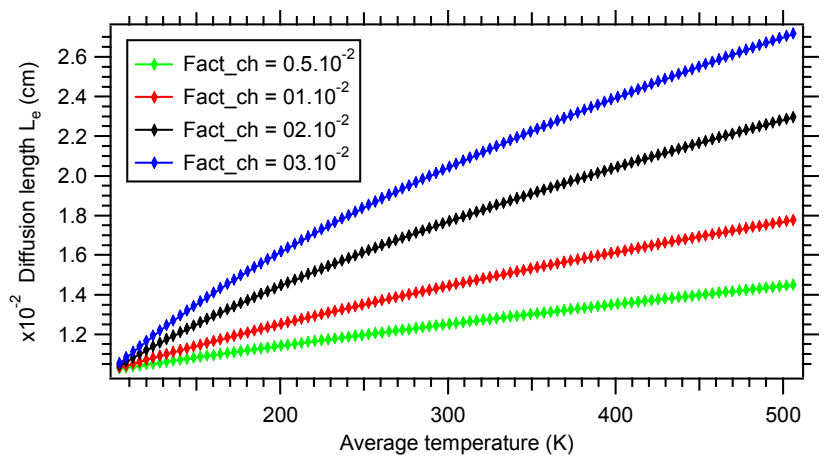

Figure 12. Electron diffusion length as a function of mean temperature for different heating factor values.

$\mathrm{n}_{\mathrm{i}}=1.4510^{10} \mathrm{~cm}^{-3 ;} \mathrm{n} \_\mathrm{mott}=1.0310^{18} \mathrm{~cm}^{-3 ;} \mathrm{Se}=\mathrm{Sx}=10 \mathrm{~cm} \mathrm{~s}^{-1} ;$ bv_max $=10^{-7} \mathrm{~cm}^{3}$ $\mathrm{s}^{-1} ; \mathrm{L}_{\mathrm{e}}=\mathrm{f}$ (mean temperature); $\mathrm{L}_{\mathrm{x}}=\mathrm{f}$ (mean temperature); $\mathrm{F}_{\mathrm{o}}=10$.

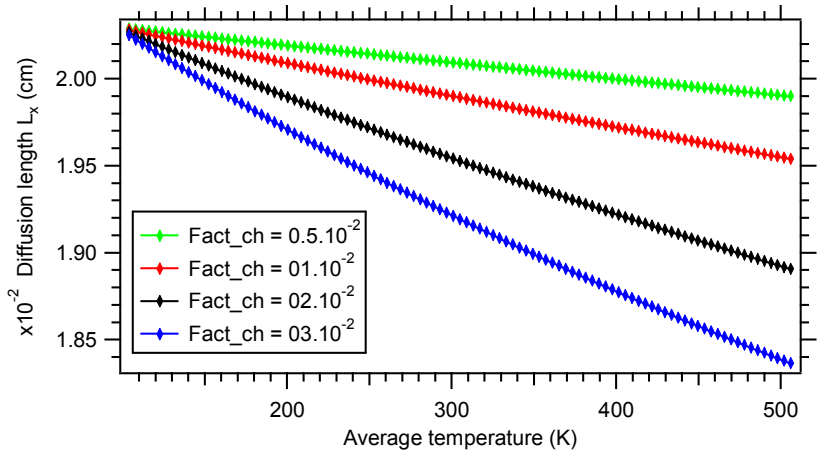

Figure 13. Exciton diffusion length as a function of mean temperature for different heating factor values.

$\mathrm{n}_{\mathrm{i}}=1.4510^{10} \mathrm{~cm}^{-3 ;} \mathrm{n} \_$mott $=1.0310^{18} \mathrm{~cm}^{-3 ;} \mathrm{Se}=\mathrm{Sx}=10 \mathrm{~cm} \mathrm{~s}^{-1} ;$ bv_max $=10^{-7} \mathrm{~cm}^{3}$ $\mathrm{s}^{-1} ; \mathrm{L}_{\mathrm{e}}=\mathrm{f}$ (mean temperature); $\mathrm{L}_{\mathrm{x}}=\mathrm{f}$ (mean temperature); $\mathrm{Fo}=0, \overline{8}$.

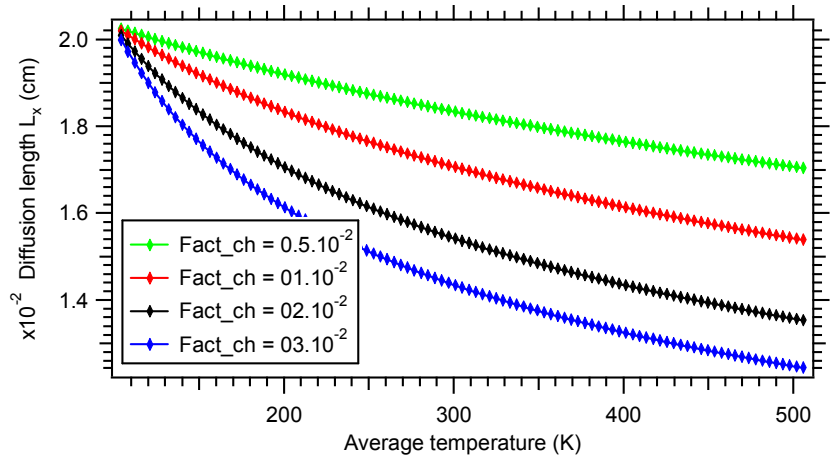

Figure 14. Exciton diffusion length as a function of mean temperature for different heating factor values.

$\mathrm{n}_{\mathrm{i}}=1.4510^{10} \mathrm{~cm}^{-3 ;} \mathrm{n} \_$mott $=1.0310^{18} \mathrm{~cm}^{-3 ;} \mathrm{Se}=\mathrm{Sx}=10 \mathrm{~cm} \mathrm{~s}^{-1} ;$ bv_max $=10^{-7} \mathrm{~cm}^{3}$ $\mathrm{s}^{-1} ; \mathrm{L}_{\mathrm{e}}=\mathrm{f}$ (mean temperature); $\mathrm{L}_{\mathrm{x}}=\mathrm{f}$ (mean temperature); $\mathrm{Fo}_{\mathrm{o}}=10$.

\section{c. Influence of the ratio between the mobility of excitons and electrons.}

The findings on the negligible effect of the mobility ratio on the variation of electron diffusion length as a function of mean temperature in Figure 4 remain the same for Figures 15 and 16. 


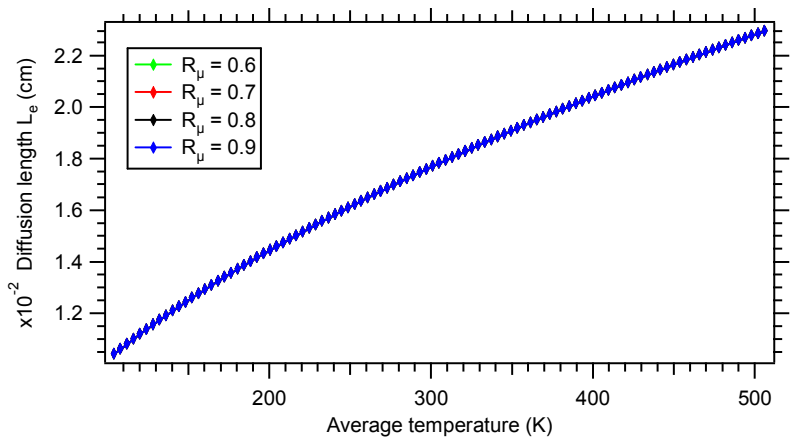

Figure 15. Diffusion length of electrons as a function of mean temperature for different values of $R \mu$.

$\mathrm{n}_{\mathrm{i}}=1.4510^{10} \mathrm{~cm}^{-3 ;} \mathrm{n} \_$mott $=1.0310^{18} \mathrm{~cm}^{-3 ;} \mathrm{Se}=\mathrm{Sx}=10 \mathrm{~cm} \mathrm{~s}^{-1} ;$ bv_max $=10^{-7} \mathrm{~cm}^{3} \mathrm{~s}^{-1}$; Fact_ch $=2.10^{-2} ; \mathrm{L}_{\mathrm{e}}=\mathrm{f}$ (mean temperature); $\mathrm{Lx}=\mathrm{f}$ (mean temperature); $\mathrm{Fo}=10$.

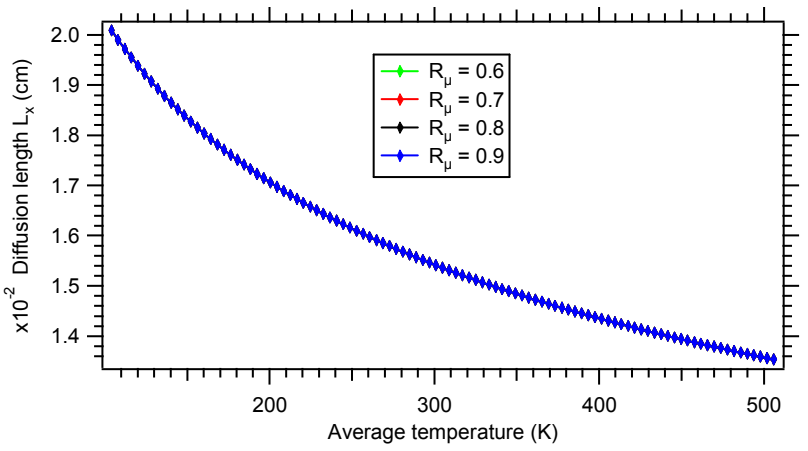

Figure 16. Exciton diffusion length as a function of mean temperature for different values of $R \mu$.

$\mathrm{n}_{\mathrm{i}}=1.4510^{10} \mathrm{~cm}^{-3 ;} \mathrm{n} \operatorname{mott}=1.0310^{18} \mathrm{~cm}^{-3 ;} \mathrm{Se}=\mathrm{Sx}=10 \mathrm{~cm} \mathrm{~s}^{-1} ; \mathrm{bv} \max =10^{-7} \mathrm{~cm}^{3}$ $\mathrm{s}^{-1} ;$ Fact_ch $=2.10^{-2} ; \overline{\mathrm{L}}_{\mathrm{e}}=\mathrm{f}$ (mean temperature); $\mathrm{L}_{\mathrm{x}}=\mathrm{f}$ (mean temperature); $\mathrm{Fo}=10$.

\subsubsection{Total Photocurrent Density as a Function of the Mean Temperature}

The curves of total photocurrent density versus mean temperature for varying electron and exciton diffusion lengths are shown in Figures 17, 18, 19 and 20.

a. Influence of the ratio between the diffusion time and the lifetime of the charge carriers.

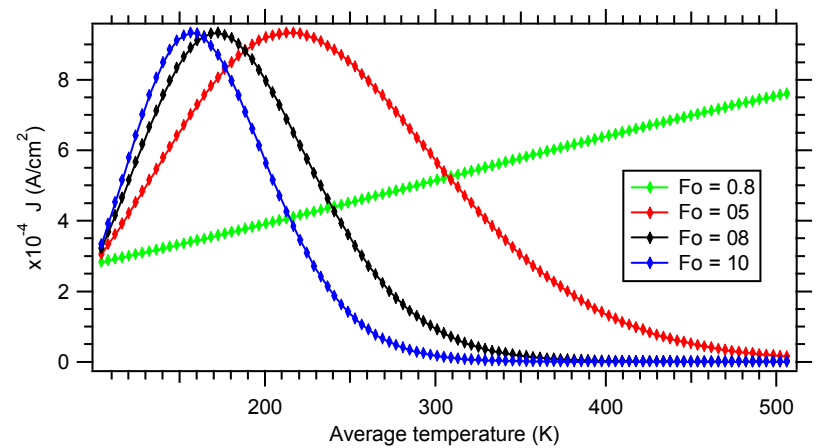

Figure 17. Total photocurrent density of charge carriers as a function of mean temperature for different values of Fo.

$\mathrm{n}_{\mathrm{i}}=1.4510^{10} \mathrm{~cm}^{-3 ;} \mathrm{n} \_$mott $=1.0310^{18} \mathrm{~cm}^{-3 ;} \mathrm{Se}=\mathrm{Sx}=10 \mathrm{~cm} \mathrm{~s}^{-1} ; \mathrm{bv} \_\max =10^{-7} \mathrm{~cm}^{3}$ $\mathrm{s}^{-1} ;$ Fact_ch $=2.10^{-2} ; \overline{\mathrm{L}}_{\mathrm{e}}=\mathrm{f}$ (mean temperature); $\mathrm{L}_{\mathrm{x}}=\mathrm{f}$ (mean temperature).
To study the influence of the Fourier number on the total photocurrent density, we fixed the heating factor and varied the diffusion lengths of the charge carriers. According to the curves in Figure 17, we have two different profiles of the total photocurrent density. The first profile corresponds to a Fourier number $\mathrm{Fo}_{\mathrm{O}}=0.8$ and the second corresponds to the large Fourier number values. For $\mathrm{Fo}=0.8$, we have an augmentation of the total photocurrent density as a function of the mean temperature. This shows that the dissociated and diffused charge carriers participate in the photocurrent. The smaller the Fourier number, the greater the diffusion length of the excitons and the greater the collected photocurrent. On the other hand, for large Fourier number values, we have maxima between $150 \mathrm{~K}$ and $250 \mathrm{~K}$ followed by a considerable decrease in total photocurrent density for mean temperatures above $250 \mathrm{~K}$. The very low values of the total photocurrent density between $250 \mathrm{~K}$ and $500 \mathrm{~K}$ can be explained by the low values of the diffusion length of the charge carriers, in particular that of the excitons and the recombination of the charge carriers.

Whereas, the maxima between $150 \mathrm{~K}$ and $250 \mathrm{~K}$ are explained by a high carrier collection rate and a high rate of dissociation of excitons by the electric field.

b. Influence of the ratio between imposed heat flux and that of thermal conduction.

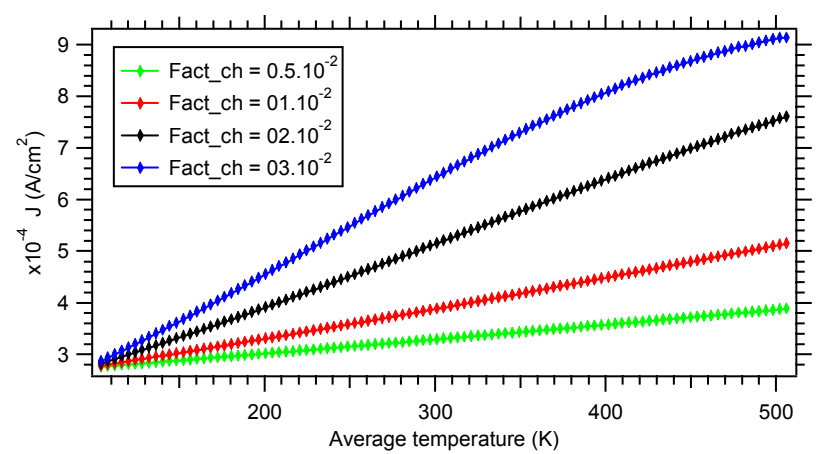

Figure 18. Total photocurrent density of charge carriers as a function of mean temperature for different heating factor values.

$\mathrm{n}_{\mathrm{i}}=1.4510^{10} \mathrm{~cm}^{-3 ;} \mathrm{n} \_$mott $=1.0310^{18} \mathrm{~cm}^{-3 ;} \mathrm{Se}=\mathrm{Sx}=10 \mathrm{~cm} \mathrm{~s}^{-1} ;$ bv_max $=10^{-7} \mathrm{~cm}^{3}$ $\mathrm{s}^{-1} ; \mathrm{L}_{\mathrm{e}}=\mathrm{f}$ (mean temperature); $\mathrm{L}_{\mathrm{x}}=\mathrm{f}$ (mean temperature); $\mathrm{Fo}_{\mathrm{O}}=0,8$.

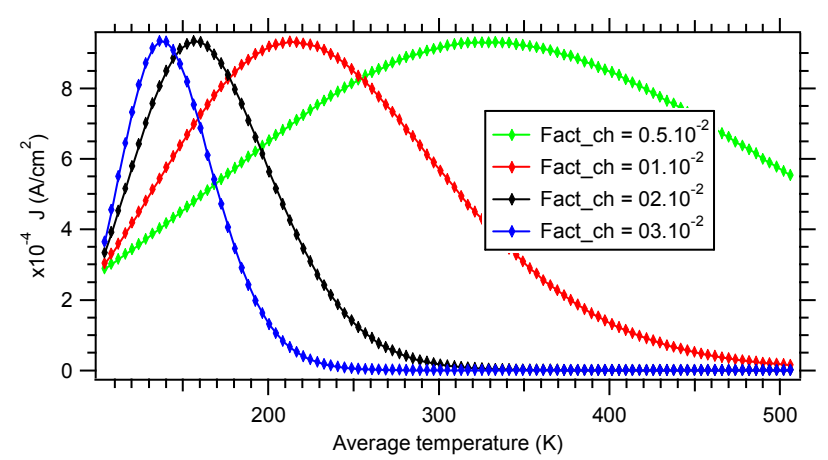

Figure 19. Total photocurrent density of charge carriers as a function of mean temperature for different heating factor values.

$\mathrm{n}_{\mathrm{i}}=1.4510^{10} \mathrm{~cm}^{-3 ;} \mathrm{n} \_\mathrm{mott}=1.0310^{18} \mathrm{~cm}^{-3 ;} \mathrm{Se}=\mathrm{Sx}=10 \mathrm{~cm} \mathrm{~s}^{-1} ; \mathrm{bv} \_\max =10^{-7} \mathrm{~cm}^{3}$ $\mathrm{s}^{-1} ; \mathrm{L}_{\mathrm{e}}=\mathrm{f}$ (mean temperature); $\mathrm{L}_{\mathrm{x}}=\mathrm{f}$ (mean temperature); $\mathrm{F}_{\mathrm{O}}=10$. 
The findings and interpretations made on the variation of total photocurrent density of charge carriers as a function of mean temperature in Figures 6 and 7 remain the same for Figure 18.

On the other hand, in Figure 19, we represent the evolution of the total photocurrent density as a function of the mean temperature for a large Fourier number value. The findings noted in this figure, that is a considerable decrease and low values of the total photocurrent density are due to the negative effects of the heating factor, Fourier number and mean temperature on the exciton diffusion length. Therefore, we have a very significant augmentation of the total photocurrent density for the low values of the heating factor, Fourier number and mean temperature.

Indeed, the photogenerated carriers in the depletion region are immediately dissociated and separated by the electric field and subsequently collected. On the other hand, those photogenerated outside this region must travel a certain distance by thermal agitation before reaching the depletion region. If this distance is less than the diffusion length of the charge carriers, in particular the excitons, the photogenerated carriers are collected and contribute to the improvement of the total photocurrent density. On the other hand, if this distance is greater than the diffusion length of the excitons, then these carriers recombine and the collection rate of the carriers decreases, thus decreasing of the total photocurrent density. The decrease of the total photocurrent density may also be due to the variation in the space charge area, as this is a function of the mean temperature.

c. Influence of the ratio between the mobility of excitons and electrons.

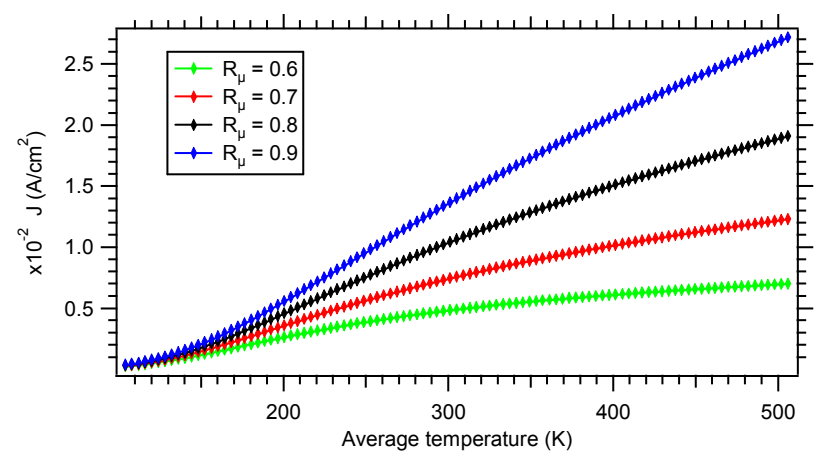

Figure 20. Total photocurrent density of charge carriers as a function of mean temperature for different values of $R \mu$.

$\mathrm{n}_{\mathrm{i}}=1.4510^{10} \mathrm{~cm}^{-3 ;} \mathrm{n} \_\mathrm{mott}=1.0310^{18} \mathrm{~cm}^{-3 ;} \mathrm{Se}=\mathrm{Sx}=10 \mathrm{~cm} \mathrm{~s}^{-1} ; \mathrm{Fo}=10 ;$ bv_max $=10^{-7} \mathrm{~cm}^{3} \mathrm{~s}^{-1} ;$ Fact_ch $=2.10^{-2} ; \mathrm{L}_{\mathrm{e}}=\mathrm{f}$ (mean temperature) $\mathrm{L}_{\mathrm{x}}=\mathrm{f}$ (mean temperature).

The findings and interpretations made on the variation of total photocurrent density of charge carriers as a function of mean temperature in Figure 8 remain the same for Figure 20.

\subsection{Total Photocurrent Density as a Function of the Ratio of Mobilities: Electron and Exciton Diffusion Lengths}

The curves of total photocurrent density versus mobility ratio for varying electron and excitation diffusion lengths are shown in Figures 21, 22 and 23. The mobility ratio $R_{\mu}=\frac{\mu_{x}}{\mu_{e}}$ is a dimensionless characteristic quantity. It is an extremely influential parameter on the total photocurrent density.

a. Influence of the ratio between diffusion time and charge carrier lifetime.

According to the results obtained previously we have an augmentation of the total photocurrent density as a function of the mean temperature for a Fourier number $\mathrm{Fo}=0.8$. While the large values of the mean temperature correspond to the small values of the mobility ratio, that is why the total photocurrent density is significant for $\mathrm{Fo}=0.8$ and for $R_{\mu} \geq 0.2$. On the other hand, always in this range the total photocurrent density is low for the large values of the Fourier number.

The maxima are between $R_{\mu}=0.2$ and $R_{\mu}=0.6$ for the Fourier number values.

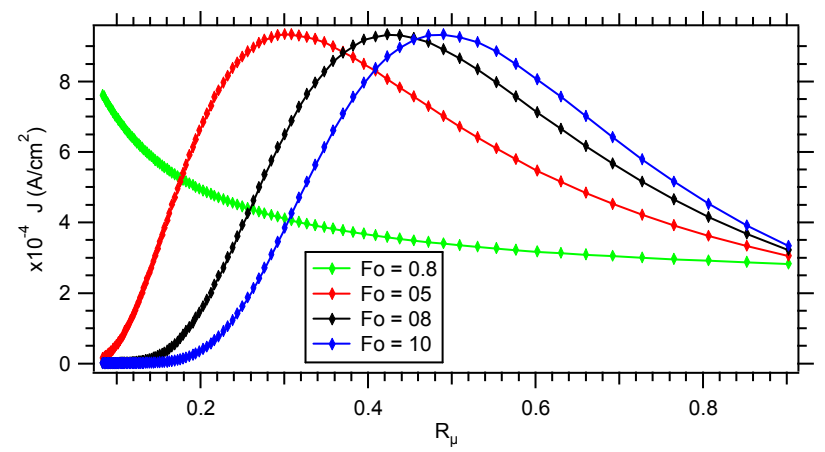

Figure 21. Total photocurrent density of charge carriers as a function of the ratio of mobilities for different values of $F o$.

$\mathrm{n}_{\mathrm{i}}=1.4510^{10} \mathrm{~cm}^{-3 ;} \mathrm{n} \_$mott $=1.0310^{18} \mathrm{~cm}^{-3} ; \mathrm{Se}=\mathrm{Sx}=10 \mathrm{~cm} \mathrm{~s}^{-1} ; \mathrm{bv} \_\max =10^{-7} \mathrm{~cm}^{3}$ $\mathrm{s}^{-1}$; Fact_ch $=2.10^{-2} ; \overline{\mathrm{L}}_{\mathrm{e}}=\mathrm{f}$ (mean temperature); $\mathrm{L}_{\mathrm{x}}=\mathrm{f}$ (mean temperature).

On the other hand, the green curve obtained for $\mathrm{Fo}=0.8$, the first instants of decay result in a rapid fall in total photocurrent density and are dominated by charge carrier diffusion; then comes a period corresponding to a slower decay and linked to photogenerated carrier recombination phenomena in the base.

The variation in the length of carrier diffusion, that is its augmentation, has a direct effect on the improvement of the photocurrent density.

b. Influence of the ratio between imposed heat flux and that of thermal conduction.

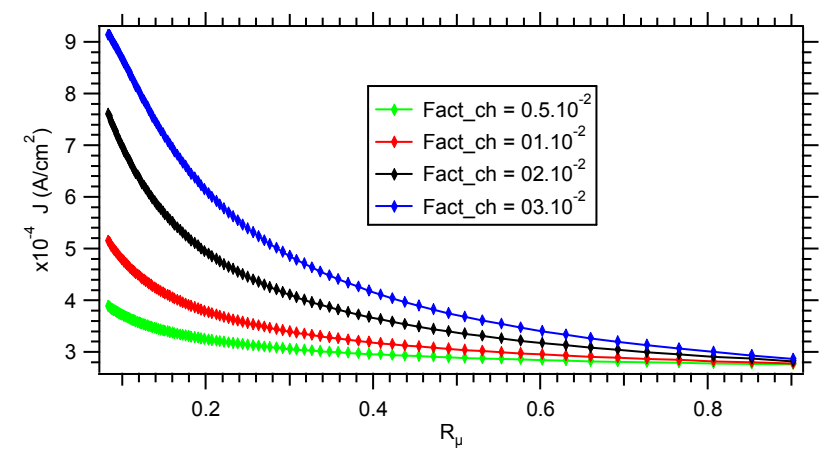

Figure 22. Total photocurrent density of charge carriers as a function of the ratio of mobilities for different values of the heating factor.

$\mathrm{n}_{\mathrm{i}}=1.4510^{10} \mathrm{~cm}^{-3 ;} \mathrm{n} \_\mathrm{mott}=1.0310^{18} \mathrm{~cm}^{-3 ;} \mathrm{Se}=\mathrm{Sx}=10 \mathrm{~cm} \mathrm{~s}^{-1} ;$ bv_max $=10^{-7} \mathrm{~cm}^{3}$ $\mathrm{s}^{-1} ; \mathrm{L}_{\mathrm{e}}=\mathrm{f}$ (mean temperature); $\mathrm{L}_{\mathrm{x}}=\mathrm{f}$ (mean temperature); $\mathrm{Fo}=0,8$. 


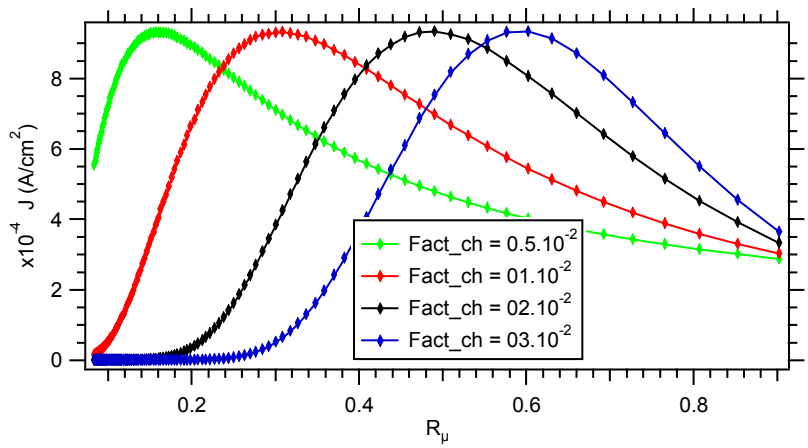

Figure 23. Total photocurrent density of charge carriers as a function of the ratio of mobilities for different values of the heating factor.

$\mathrm{n}_{\mathrm{i}}=1.4510^{10} \mathrm{~cm}^{-3 ;} \mathrm{n} \_$mott $=1.0310^{18} \mathrm{~cm}^{-3 ;} \mathrm{Se}=\mathrm{Sx}=10 \mathrm{~cm} \mathrm{~s}^{-1} ; \mathrm{bv} \_\max =10^{-7} \mathrm{~cm}^{3}$ $\mathrm{s}^{-1} ; \mathrm{L}_{\mathrm{e}}=\mathrm{f}$ (mean temperature); $\mathrm{L}_{\mathrm{x}}=\mathrm{f}$ (mean temperature); $\mathrm{Fo}=10$.

The findings and interpretations made on the variation of the total photocurrent density of the charge carriers as a function of the mobility ratio in Figure 21 for $R_{\mu} \geq 0.2$ the maxima between $R_{\mu}=0.2$ and $R_{\mu}=0.6$ remain the same for Figures 22 and 23.

\section{Conclusion}

In conclusion, we studied the evolution of the total photocurrent density as a function of the mean temperature and its evolution as a function of the mobility ratio of a silicon-based semiconductor. We first studied the effects of three admittance parameters on the total photocurrent density for an excitation diffusion length $L_{x}=3 \cdot 43 \cdot 10^{-2} \mathrm{~cm}$. It appears from this study that all these parameters have a positive effect on the total photocurrent density.

In addition, we studied the effects of these parameters on the total photocurrent density for variable charge carrier diffusion lengths.

The phenomena of excitation dissociation depend on the electric field of the carrier diffusion length. Whereas, those of recombination depend on the carrier diffusion length and the thickness of the semiconductor.

It appears from our study that the influence of the heating factor and the Fourier number on the total photocurrent density is more considerable with small values of the Fourier number. The positive influence of the ratio of mobilities on the total photocurrent density is more considerable with a diffusion length of the excitons $L_{x}=3 \cdot 43 \cdot 10^{-2} \mathrm{~cm}$.

In order to develop a numerical model of heat-bearing solar cells, it is recommended to consider an exciton diffusion length $L_{x}=3.43 .10^{-2} \mathrm{~cm}$ longer than that of electrons. For this purpose, it is also recommended to work with small Fourier number values.

\section{References}

[1] M. Burgelman, B. Minnaert; Including excitons in semiconductor solar cell modeling. Thin Solid Films 511-512, 214-218 (2006).
[2] S. Zh. Karazhanov; Temperature and doping level dependence solar cell performance Including excitons. Solar Energy Materials \& Solar Cells 63 (2000) 149-163.

[3] R. Corkish, D. S. P. Chan, and M. A. Green; Excitons in silicon diodes and solar cells: A three-particle theory. Institute of Physics. [(S0021-8979 (1996) 0070-9].

[4] M. Niane, S. Ndiaye, M. Faye, M. Pilor, M. Diagne, N. Mbengue, O. A. Niasse, B. Ba; Variation of Excess Excitons Density in Function to Silicon Solar Cell Parameters; Journal of Materials Science \& Surface Engineering; volume 4 (6) (2016) 448-451.

[5] M. Faye, M. MBow, M. Ba: Numerical Modeling of the Effects of Excitons in a Solar Cell Junction $n+p$ of the Model by Extending the Space Charge Layer; International Review of Physics (I. RE. PHY), Vol. 8, N. 4 ISSN 1971-680X (August 2014).

[6] M. Faye, M. Niane, S. Ndiaye, O. Ngom, C. Mbow, B. Ba, Numerical Modelling of Effects of Excitons on Photoelectric Properties of Cells; Journal of Scientific and Engineering Research; volume 6 (6) (2019) 138-146.

[7] M. Faye, C. Mbow, B. Ba; Internal Electric Field In The Space Charge Layer Of A Solar Cell Based On Silicon In The Presence Of Excitons; International journal of scientific \& technology research; volume 4 (2015) 66-69.

[8] M. Faye, M. Niane, S. Ndiaye, C. Mbow, B. Ba; Effects of Variability of The Average Temperature on The Distribution of Electrons and of Excitons in A Semiconductor; Journal of Materials Science \& Surface Engineering; volume 4 (7) (2016) 467-471.

[9] M. Faye, S Ndiaye, C. MBow, B. Ba; Effects of the Average Temperature on the Photocurrent Density of Inorganic Solar Cells Based on Silicon in the Presence of Excitons, International Journal of Engineering Trends and Technology (IJETT); Volume 24 Number 3- June 2015 E-ISSN 2231-5381, P-ISSN: 2349-0918.

[10] M. Faye, M MBow, M Ba: Development a Numerical Model Applicable to Inorganic and Organic Solar Cells Based on Silicon in the Presence of Excitons; Current Trends in Technology and Science, ISSN: 2279-0535; Volume 04, Issue 02 (Feb - Mar. 2015).

[11] O. Ngom, M. Faye, M. Mbaye, C. Mbow, B. Ba; Numerical Study of the Effect of Temperature on the Performance of a Silicon Heterojunction Solar Cell (HIT) in the Presence of Excitons; International Journal of Materials Science and Applications; volume 8 (4) (2019) 56-67.

[12] R. B. Bird, W. E. Stewart, E N. Lightfoot: Transport Phenomena, John Wiley and Sons, Inc, New York 2001.

[13] S. V. Patankar: "Numerical Heat Transfer and Fluid Flow", Hemisphere Publishing Corporation, McGraw-Hill Book Company, 1981.

[14] D. W. Peaceman, H. A. Rachford, The Numerical Solution of Parabolic and Elliptic Difference Equations, J. Soc. Ind., Appli. Math, 3, 28-43, 1955.

[15] Ji-Ting Shieh, Chiou-Hua Liu, Hsin-Fei Meng, Shin-Rong Tseng, Yu-Chiang Chao, and Sheng-Fu Horng; The effect of carrier mobility in organic solar cells. Journal of Applied Physics 107, 0845032010. 